\title{
Dieta da paca (Cuniculus paca) usando métodos indiretos numa área de cultura agrícola na Floresta Atlântica brasileira
}

\author{
Rodrigo Zucaratto ${ }^{1^{*}}$ \\ Renata Carrara ${ }^{2}$ \\ Brena Karina Siqueira Franco ${ }^{2}$ \\ ${ }^{1}$ Laboratório de Ecologia e Conservação de Populações, Departamento de Ecologia \\ Instituto de Biologia, CCS, Universidade Federal do Rio de Janeiro \\ Caixa Postal 68020, CEP 21941-590, Rio de Janeiro - RJ, Brasil \\ ${ }^{2}$ Escola Superior São Francisco de Assis (ESFA) \\ Rua Bernadino Monteiro, 700, CEP 29650-000, Santa Teresa - ES, Brasil \\ *Autor para correspondência \\ rzucarattobio@gmail.com
}

\section{Resumo}

A paca (Cuniculus paca) é um roedor que se alimenta de frutos de acordo com sua disponibilidade. Este estudo descreve o consumo de frutos por pacas numa área de cultivo sombreado de cacau na Floresta Atlântica do Espírito Santo. Foi realizada a procura de vestígios indiretos deixados por estes animais, como as marcas características dos incisivos, para reconhecer os frutos consumidos. Doze espécies foram consumidas pelas pacas, pertencentes a 10 famílias e 10 gêneros. Essa lista inclui seis espécies nativas e seis espécies exóticas. Alguns frutos foram consumidos inteiros, enquanto que outros tiveram seu exocarpo ou suas sementes descartadas. Os métodos indiretos mostraram-se adequados para caracterizar a dieta dessa espécie e podem complementar os métodos de pesquisa convencionais tais como visualização direta e análises de conteúdo estomacal ou fecal. A ocorrência das pacas na área de cultivo demonstra plasticidade quanto à sua dieta e a possibilidade de conservação de populações dessa espécie em áreas alteradas fora das unidades de conservação.

Unitermos: dispersão de sementes, frugivoria, marca de incisivos, roedores

\section{Abstract}

Diet of paca (Cuniculus paca) using indirect methods in an agricultural area in the Brazilian Atlantic Forest. The paca (Cuniculus paca) is a rodent that feeds on fruits according to their availability. This study describes the consumption of fruit by paca in an area of shaded cultivation of cocoa in the Brazilian Atlantic Forest. The study was carried out by the search for tracks left by these animals, such as marks of incisors found in fruits, in order to recognize the fruits consumed. We recorded 12 species consumed by the pacas, belonging to 10 families and 10 genera. The list included six native species and six exotic species. Some fruits were eaten as a whole, while others had their exocarp or seeds discarded. The indirect methods showed suitability to characterize the diet of this species, and they could complement conventional research methods such as direct sighting and analyses of stomachal or fecal contents. The occurrence of pacas in the agricultural area shows the plasticity of their diet and the possibility of conserving populations of this species in disturbed areas outside nature reserves.

Key words: frugivory, incisor marks, rodents, seed dispersal 
A paca (Cuniculus paca) é uma espécie noturna e distribui-se do sul do México ao norte da Argentina. Ela ocupa preferencialmente florestas tropicais úmidas, contudo ocorre numa variedade de habitats, incluindo manguezais, florestas decíduas, semidecíduas e usualmente procuram áreas florestadas próximas a cursos de água. Forrageia ao entardecer e no crepúsculo se deslocando por trilhas fixas e próprias de cada indivíduo, que os levam diretamente aos locais de alimentação (Perez, 1992). A paca é considerada generalista quanto à dieta e alimenta-se principalmente de frutos disponíveis no decorrer das estações (Perez, 1992; Beck-King et al., 1999). Os dentes frontais das pacas crescem permanentemente e o animal os desgasta roendo madeira ou objetos duros (Perez-Torres, 1996). Elas são consideradas dispersoras de sementes pequenas (Beck-King et al., 1999; Janini, 2000) e predadoras das grandes sementes (Janini, 2000). No entanto, podem eventualmente atuar como dispersoras dessas grandes sementes, uma vez que se afastam dos locais de alimentação carregando os frutos e não consomem todas as sementes carregadas.

A dispersão de sementes é importante na manutenção e regeneração das florestas tropicais (Terborgh e Wright, 1994), dependendo em grande parte de roedores como cutias (Dasyprocta spp.) e pacas. Estudos recentes, no entanto, tem demonstrado que esses animais são negativamente afetados pelo processo de fragmentação (Galetti et al., 2006; Jorge e Howe, 2009), comprometendo a regeneração das plantas dispersadas pelos mesmos. Esse processo vem ocorrendo intensamente na Floresta Atlântica, que tem uma longa história de exploração madeireira, culturas agrícolas e, mais recentemente, plantação de eucalipto (Galindo-Leal e Câmara, 2003).

Ao contrário do que se conhece a respeito da dieta de outros mamíferos neotropicais, como pequenos roedores, primatas e ungulados, a dieta de grandes roedores como a paca tem sido pouco investigada (Dubost e Henry, 2006). Estudos sobre a dieta de pacas, principalmente em ambientes que sofreram ação antrópica, deveriam ser realizados para subsidiar estratégias de conservação dessa espécie em áreas fora das unidades de conservação. Dessa forma, os objetivos deste estudo foram: 1) identificar os frutos utilizados por pacas em uma área de cultura agrícola na Floresta Atlântica; 2) avaliar a aplicabilidade de métodos indiretos, neste caso marcas de incisivos deixadas em frutos, para determinar a dieta da espécie.

O estudo foi realizado na Fazenda Sete Quedas, uma propriedade rural localizada no município de João Neiva (1945'28'S, 40²3'09'W), sudeste do Brasil. A área estudada é destinada ao cultivo de culturas agrícolas como cacau (Theobroma cacao) e eucalipto (Eucalyptus spp.). Próximo à fazenda existe um fragmento de Floresta Atlântica Submontana de formação primária com aproximadamente 30ha que é cercado por outros remanescentes florestais. Há na fazenda um pequeno riacho atravessando a área de cultivo. A temperatura média anual é de $35^{\circ} \mathrm{C}$ e a precipitação anual média é de $1200 \mathrm{~mm}$.

Para identificar os frutos consumidos pelas pacas foram realizados levantamentos semanais em busca das espécies vegetais em frutificação e foi usada também uma armadilha fotográfica para registrar se as pacas consumiam frutos da área, quando esses foram deixados no chão como ceva. Esses levantamentos ocorreram de junho a novembro de 2008, consistindo numa busca aleatória pelas espécies vegetais que apresentavam frutos em toda a área de cultivo agrícola. Cada espécie vegetal encontrada foi identificada por meio de bibliografia pertinente (Barroso et al., 1999; Lorenzi, 2002; Missouri Botanical Garden, 2008) e por comparação com material do herbário da Universidade Federal do Espírito Santo, realizando-se consulta a especialistas quando necessário. Para verificar se os frutos foram consumidos por pacas, foi realizada uma busca por vestígios indiretos deixados por estes animais, como as marcas características dos incisivos e trilhas. A análise das marcas de incisivos segue o recomendado por Beck-King et al. (1999), que consideraram que marcas maiores que quatro milímetros de largura para cada incisivo devem pertencer à paca adulta, quando comparado com cutias (Dasyprocta spp.). A armadilha fotográfica foi disposta em dois pontos distantes $1 \mathrm{~km}$ um do outro, permanecendo durante quinze dias em cada ponto, monitorando continuamente e sendo verificadas a cada três dias. Esses pontos foram escolhidos por possuírem indícios da presença das pacas. 
Um ponto foi localizado dentro da área cultivada e outro numa área de pastagem. Em cada ponto foi montada uma ceva com jaca (Artocarpus heterophyllus), com a finalidade de atrair os animais.

Foram obtidos dois registros fotográficos confirmando a presença das pacas na área de estudo, um em cada ponto de amostragem. Dentre as 14 espécies vegetais encontradas produzindo frutos, 12 foram utilizadas como recurso alimentar pelas pacas. Os frutos das espécies consumidas distribuíram-se em 10 famílias e 10 gêneros, sendo seis espécies nativas e seis espécies exóticas (Tabela 1 e Figura 1). Aroeira-vermelha (Schynus terebentifolius) e manga (Mangifera indica) não apresentaram vestígios que pudessem incluí-las na dieta das pacas. Para as espécies, Citrus x sinensis, Citrus limon, Citrus deliciosa e Artocarpus heterophyllus, tanto o endocarpo quanto as sementes, com exceção do tegumento, foram consumidos. Em diversas ocasiões foram encontrados troncos de Myrtaceae, possivelmente goiabeira (Psidium guajava) ou araçá (Psidium sp.), apresentando marcas de incisivos.

A utilização das marcas de incisivos é recomendada para descrever a dieta das pacas (Beck-King et al., 1999) por tratar-se de um método que dispensa a visualização direta dessa espécie que ocorre em baixa densidade e possui hábitos discretos e noturnos. Apesar disso, Beck-King et al. (1999) questionaram a confiabilidade deste método, quando se compara as marcas deixadas por cutias (Dasyprocta spp.) com aquelas feitas pelas pacas juvenis, por serem bastante semelhantes. Contudo, esses autores confirmam que marcas acima de quatro milímetros podem ser atribuídas às pacas adultas. Uma outra espécie que poderia deixar marcas de incisivos iguais ou maiores que o esperado por pacas é a capivara (Hidrochaeris hidrochaeris). No entanto, as marcas de incisivos encontradas em nosso estudo não deveriam ser atribuídas a essas outras espécies, pois durante todo período amostral nenhum indício da presença de cutias ou capivaras foi encontrado e não há registros históricos da ocorrência dessas espécies na área de estudo. Por diversas vezes foram encontrados troncos de Myrtaceae com marcas de incisivos de pacas. Os dentes frontais desses animais crescem permanentemente (Perez, 1992; Perez-Torrez, 1996) e de acordo com alguns autores (Perez-Torrez, 1996; Hosken, 1999) é necessário oferecer às pacas em cativeiro galhos de goiabeira para que possam desgastar os dentes. Sendo assim, pressupõe-se que o comportamento descrito acima possui a finalidade de desgaste dos dentes.

TABELA 1: Itens alimentares consumidos por pacas na Fazenda Sete Quedas. Origem: (N) espécie nativa, (E) espécie exótica; Tipo de Vestígio: (I) Marca de incisivo, (T) Trilha característica; Consumo: (FI) Fruto inteiro, (M) mesocarpo, (E) endocarpo, (S) semente.

\begin{tabular}{lllccc}
\hline Família & Espécie & Nome comum & Origem & Tipo de Vestígio & Consumo \\
\hline Lecythidaceae & Lecythis lanceolata & Sapucaia & $\mathrm{N}$ & $\mathrm{I}, \mathrm{T}$ & $\mathrm{S}$ \\
Flacourtiaceae & Carpotroche brasiliensis & Sapucainha & $\mathrm{N}$ & $\mathrm{I}, \mathrm{T}$ & $\mathrm{S}$ \\
Anacardiaceae & Spondias mombin & Cajá-mirim & $\mathrm{N}$ & $\mathrm{T}$ & $\mathrm{FI}$ \\
Sterculiaceae & Theobroma cacao & Cacau & $\mathrm{N}$ & $\mathrm{I}, \mathrm{T}$ & $\mathrm{S}$ \\
Euphorbiaceae & Cnidoscolus pubescens & Arrediabo & $\mathrm{N}$ & $\mathrm{I}$ & $\mathrm{S}$ \\
Passifloraceae & Passiflora sp. & Maracujá do mato & $\mathrm{N}$ & $\mathrm{T}$ & $\mathrm{S}$ \\
Rutaceae & Citrus $x$ sinensis & Laranja & $\mathrm{E}$ & $\mathrm{T}$ & $\mathrm{E}, \mathrm{S}$ \\
Rutaceae & Citrus limon & Limão & $\mathrm{E}$ & $\mathrm{T}$ & $\mathrm{E}, \mathrm{S}$ \\
Rutaceae & Citrus deliciosa & Mexirica & $\mathrm{E}$ & $\mathrm{T}$ & $\mathrm{E}, \mathrm{S}$ \\
Moraceae & Artocarpus heterophyllus & Jaca & $\mathrm{E}$ & $\mathrm{T}$ & $\mathrm{E}, \mathrm{S}$ \\
Musaceae & Musa parasidiaca & Banana & $\mathrm{E}$ & $\mathrm{I}$ & $\mathrm{E}$ \\
Lauraceae & Persea americana & Abacate & $\mathrm{E}$ & $\mathrm{I}$ & $\mathrm{M}, \mathrm{E}, \mathrm{S}$ \\
\hline
\end{tabular}



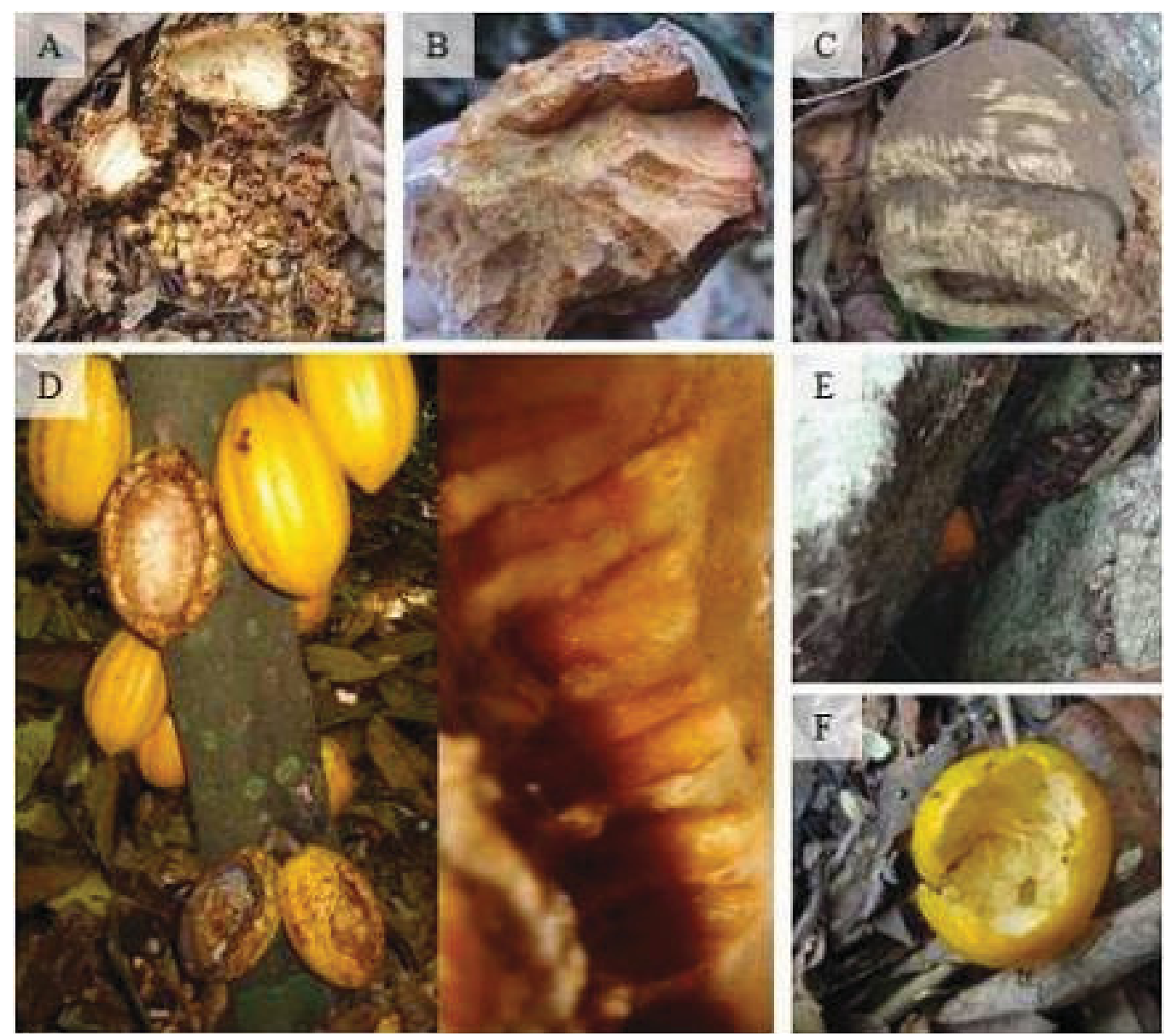

FIGURA 1: Marcas de incisivos de Cuniculus paca encontradas em alguns frutos na Fazenda Sete Quedas. (A) Sapucainha - Carpotroche brasiliensis; (B) Abacate - Persea americana, semente; (C) Sapucaia - Lecythis lanceolata; (D) Cacau - Theobroma cacao; (E) Mexirica - Citrus deliciosa na entrada da toca e (F) Mexirica - Citrus deliciosa.

As sementes de sapucaia (Lecythis lanceolata) foram consumidas quando caíam ao chão. Porém, houve casos onde foram encontrados alguns frutos derrubados (ainda verdes) apresentando marcas de incisivos exatamente no opérculo por onde são liberadas as sementes do fruto, mostrando que as pacas forçaram a abertura para retirar a semente. Jannini (2000) menciona que as pacas não têm a mesma habilidade das cutias para quebrar sementes duras, dando preferência às polpas. No entanto, foi observado o consumo das sementes de sapucaia e cajá-mirim (Spondia mombin) que possuem tegumento extremamente rígido. Além disso, as sementes de cajá-mirim já foram reportadas na dieta de pacas (Beck-King et al., 1999).

Apesar das marcas de incisivos deixadas na polpa de jaca (Artocarpus heterophyllus) não terem sido claras, esta espécie foi incluída na dieta das pacas pelas marcas encontradas na casca e nas sementes. Além disso, os animais foram registrados pela armadilha fotográfica se alimentando nas cevas preparadas com jaca. $\mathrm{O}$ consumo de espécies exóticas também foi observado nos trabalhos de Beck-King et al. (1999) e Janini (2000). Apesar da manga ter sido descrita na dieta de pacas por Beck-King et al. (1999), não foram encontrados sinais característicos que pudessem incluí-la no repertório alimentar das pacas neste estudo.

Assim como constatado por Beck-King et al. (1999), também foi observado o consumo de cacau (Theobroma cacao) pelas pacas. Os frutos do cacau foram consumidos na maioria das vezes diretamente na planta mãe, pois foram encontrados roídos, apresentando marcas características de incisivos, a uma altura de 
60 a $70 \mathrm{~cm}$ no tronco, a partir do solo. Para os demais frutos não foi observado nenhum padrão de consumo e os mesmos foram incluídos na dieta por apresentarem marcas deixadas pelos incisivos e por estarem próximos a trilhas características que levavam as pacas aos locais de alimentação.

A realização deste estudo indica que o uso de sistemas agroflorestais pode favorecer a permanência de populações de pacas em pequenos fragmentos localizados em áreas agrícolas. A presença das pacas na área de cultivo e o consumo de espécies exóticas são comprovados pelas marcas de incisivos deixadas nos frutos e pelas trilhas características encontradas. Não foi confirmado se a população ali existente estava vivendo somente na área agroflorestal ou fazia uso dela unicamente para se alimentar. Nesse último caso os animais teriam que atravessar uma grande área de pastagem para voltar aos fragmentos. No entanto, devido a grande quantidade de vestígios encontrados na área agrícola, a presença de tocas e a baixa pressão de caça no local sugerem que essa área poderia prover habitat suficiente para manter os indivíduos de paca independente da proximidade do fragmento florestal.

\section{Agradecimentos}

A Lourival Folly por permitir a realização deste estudo em sua propriedade. Ao Tiago Torri pelo apoio logístico indispensável. A Alexandra Pires, Bruno Cid, Fernando Fernandez, Leandro Macedo, Luiz Gustavo Oliveira-Santos e Pâmela Antunes pelas contribuições ao manuscrito.

\section{Referências}

Barroso, G. M.; Morim, M. P.; Peixoto, A. L.; Ichaso, C. L. F. 1999. Frutos e sementes: Morfologia aplicada à sistemática de Dicotiledôneas. Editora UFV, Viçosa, Brasil, 443pp.

Beck-King, H.; Helversen, O.; Beck-King, R. 1999. Home range, population density, and food resources of Agouti paca (Rodentia: Agoutidae) in Costa Rica: A study using alternative methods. Biotropica, 31: 675-685.

Dubost, G.; Henry, O. 2006. Comparison of diets of the accouchy, agouti and paca, the three largest terrestrial rodents of French Guianan forest. Journal of Tropical Ecology, 22: 641-651.

Galetti, M.; Donatti, C. I.; Pires, A. S.; Guimarães, P. R.; Jordano, P. 2006. Seed survival and dispersal of an endemic Atlantic forest palm: the combined effects of defaunation and forest fragmentation. Botanical Journal of the Linnean Society, 151:141-149.

Galindo-Leal, C.; Câmara, I. G. 2003. Atlantic Forest hotspots status: An overview. In: Galindo-Leal, C. \& Câmara, I. G. (Eds). The Atlantic Forest of South America: Biodversity status, threats, and outlook. Island Press, New York, USA, p.3-11.

Hosken, F. M. 1999. Criação de pacas. SEBRAE - MT, Cuiabá, Brasil, 178pp.

Jannini, A. E. 2000. Dieta e densidade populacional de Agouti paca (Rodentia, Agoutidae) em Floresta Semidecídua do leste do Brasil. Tese de Doutorado, Universidade de São Paulo, Brasil, 110pp.

Jorge, M. L. S. P.; Howe, H. F. 2009. Can forest fragmentation disrupt a conditional mutualism? A case from central Amazon. Oecologia: In press.

Lorenzi, H. 2002. Árvores brasileiras: Manual de identificação e cultivo de plantas arbóreas do Brasil. v.1 e 2. $2^{\mathrm{a}}$ ed. Editora Plantarum, Nova Odessa, Brasil, 368pp.

Missouri Botanical Garden. 2008. Plant information. Diponível em <http: www.mobot.org>. Acesso em 20 de novembro de 2008.

Perez, E. M. 1992. Agouti paca. Mammalian Species, 404: 1-7.

Perez-Torres, J. 1996. Guia para el manejo y cria de la "paca" (Agouti paca). Editora Guadalupe Ltda, Santafe de Bogotá, Colombia, 37pp.

Terborgh, J.; Wright, J. 1994. Effects of mammalian herbivores on plant recruitment in two Neotropical Forests. Ecology, 75: 18291833. 